\title{
Politique commerciale extérieure : quel rôle pour les assemblées parlementaires ? Le cas des États-Unis et de l'Union Européenne
}

\begin{abstract}
Vincent Bricart $^{1}$
\section{Résumé}

Depuis le début des années 2010, une série de nouveaux accords ou de processus de négociations commerciaux entre de grands blocs géographiques ont émergé dans le monde. L’Union Européenne (UE) et les États-Unis ont à ce titre joué un rôle de premier plan dans ces initiatives et dans leurs proliférations. Face à l'impact grandissant de la globalisation sur le quotidien des citoyens, les assemblées législatives tendent à s'impliquer progressivement dans ces processus diplomatiques et à gagner en importance et en visibilité dans leur fonction vis-à-vis de ces accords. Si bien qu'aujourd'hui, toute personne s'intéressant de près ou de loin à l'étude des accords commerciaux ne peut plus ignorer le rôle qu'y jouent les parlements des États impliqués dans ces processus. Les négociations du Partenariat Transatlantique de Commerce et d'Investissement (TTIP), de l'Accord Économique et Commercial Global (CETA) et de l'Accord de Partenariat Transpacifique (TPP) représentent ainsi de bons indicateurs du rôle, des atouts et des limites que les interventions d'un parlement dans un processus de négociation peuvent rencontrer en fonction du contexte politique ou institutionnel et de l'appui de l'opinion publique. Le Parlement européen et le Congrès américain constituent respectivement des assemblées législatives relativement puissantes, représentant un nombre de citoyens conséquent et ayant d'importantes prérogatives constitutionnelles ou institutionnelles en matière commerciale, mais évoluant dans des cadres significativement différents. Via leurs outils institutionnels et à travers des pressions formelles et informelles de la part de ces deux pouvoirs législatifs, les exécutifs se retrouvent souvent contraints de prendre en compte certaines revendications des parlementaires faisant voix aux revendications d'une partie de la société civile. À défaut, le risque de voir une négociation échouer faute de ratification de l'accord par le pouvoir législatif est important. Néanmoins, l'influence du pouvoir législatif n'est pas la même pour chaque négociation et le contexte de celle-ci va dès lors jouer un rôle important pour en déterminer l'amplitude et la visibilité d'une assemblée dans ces différents processus.
\end{abstract}

Mots-clés : Accords-commerciaux ; UE ; États-Unis ; TTIP ; TPP ; CETA

\footnotetext{
${ }^{1}$ Vincent Bricart est assistant-doctorant. Diplômé d'un master en Science Politique à l'Université de Liège (ULiège) en Belgique, il travaille actuellement comme chercheur au sein du Center for International Relations Studies (CEFIR).
} 


\section{Introduction}

Au cours de la décennie qui vient de s'écouler, on a pu voir s'accroître un phénomène qui n'est pas neuf, mais qui se veut de plus en plus fréquent dans les relations diplomatiques qu'entretiennent les démocraties libérales occidentales entre elles : la mise en place de vastes accords commerciaux de libre-échange. En une dizaine d'années, on a pu voir émerger toute une série de nouveaux accords ou de processus de négociations entre de grands blocs géographiques. L'augmentation de manière répétée de ces accords s'inscrit dans des stratégies tant à buts économiques que politiques pour les démocraties occidentales qui cherchent à s'adapter à la mondialisation et à y faire face. Parallèlement à ces accords, on a pu également constater un fait relativement nouveau dans les relations internationales post-guerre froide de ces mêmes États : l'implication croissante des assemblées parlementaires et de leurs membres dans le processus de négociation et de conclusion des traités commerciaux internationaux.

De manière générale, l'étude des relations internationales se base en premier lieu sur l'analyse des interactions entre les différents exécutifs ainsi qu'entre leurs corps diplomatiques respectifs. L'examen du rôle des assemblées parlementaires dans les relations internationales est dès lors resté pendant longtemps minoritaire parmi les chercheurs car ces institutions ont des compétences et responsabilités en matière de politique extérieure à priori plus restreintes que les autres pouvoirs de par leur vocation à traiter en premier lieu des matières internes à un État. Ainsi, les rares attributions dont elles disposent dans ce domaine se limitent principalement à une activité de contrôle et d'avalisation de l'action de l'exécutif plutôt qu'à une intervention concrète dans les relations internationales avec les autres Nations. Cependant, depuis quelques années, on assiste à un regain d'intérêt des analystes politiques pour l'étude du rôle des parlementaires sur les affaires extérieures de leur État. Face à l'impact grandissant de la globalisation sur la vie quotidienne, les assemblées législatives tendent ainsi à s'impliquer progressivement dans les processus diplomatiques avec parfois des conséquences importantes pour ces derniers. À ce titre, les accords internationaux constituent l'exemple le plus évident où la participation parlementaire se montre importante et où son impact est le plus visible.

La présente analyse aura dès lors pour objectif de démontrer comment cette influence se manifeste aujourd'hui à travers plusieurs terrains d'étude. D'une part, l'Union européenne (UE) et le Parlement européen seront analysés à travers les négociations du Partenariat Transatlantique de Commerce et d'Investissement ou en anglais Transatlantic Trade and Investment Partnership (TTIP) et de l'Accord Économique et Commercial Global (CETA), d'autre part les États-Unis et le Congrès américain seront étudiés à travers le prisme des pourparlers du TTIP et de l'Accord de Partenariat Transpacifique ou en anglais Trans-Pacific Partnership (TPP). L’UE et les États-Unis ont été choisis ici car les deux entités politiques figurent parmi les plus grands espaces économiques et démocratiques du monde. Par ailleurs, elles disposent d'assemblées législatives relativement puissantes, représentant un nombre de citoyens conséquent et ayant d'importantes prérogatives constitutionnelles en matière commerciale. Elles ont cependant une organisation, des compétences, une histoire et un mode de fonctionnement différent qui rendent l'analyse comparative particulièrement pertinente. Le choix d'analyser les accords commerciaux de manière générale se base quant à lui principalement sur le fait que ces derniers constituent des processus diplomatiques intenses, prenant place sur une certaine durée, ayant un impact important sur la vie quotidienne des citoyens et surtout dans lesquels les assemblées législatives ont le plus de marge de manœuvre en politique étrangère. Enfin, les cas spécifiques étudiés ont été sélectionnés pour leurs natures et caractéristiques similaires ainsi que pour la concomitance ou proximité de leurs différentes phases de négociation. 


\section{Tour d'horizon des compétences respectives en matière de politique étrangère a. Caractéristiques structurelles : Le Parlement européen versus le Congrès américain}

Avant de passer au cœur du sujet de cette recherche, il est utile de réaliser une comparaison entre deux caractéristiques structurelles du Parlement européen et du Congrès américain : à savoir, le «droit d'initiative législative » et les «commissions parlementaires ». Aux États-Unis, les parlementaires américains sont les seuls à pouvoir initier une proposition législative ce qui a pour conséquence de pousser le président à négocier avec les parlementaires pour qu'une loi soit introduite. Cela donne de facto un pouvoir et une influence importante à ces derniers. ${ }^{1}$ Dans 1 'UE, ce droit d'initiative parlementaire appartient exclusivement à la Commission européenne et non pas aux parlementaires. Néanmoins, le Parlement européen a le pouvoir de demander à la Commission de soumettre une proposition de « loi », sous la forme d'une directive ou d'un règlement. Par ailleurs, le pouvoir de codécision du Parlement européen ayant été considérablement accru depuis la réforme du Traité de Lisbonne, il dispose de la possibilité de refuser ou d'amender la très grande majorité des législations européennes ce qui le rend de facto beaucoup plus influent sur le devenir des législations ${ }^{2}$. Enfin, tout acte législatif doit faire l'objet d'un accord entre le Parlement et le Conseil. Dès lors, la capacité d'influence des deux assemblées, américaine et européenne, sur l'exécutif est à priori similaire ${ }^{3}$.

Parallèlement à cela, les commissions parlementaires, ou «committees » en anglais, jouent un rôle fondamental dans les deux institutions pour la rédaction de rapports d'enquête, d'analyse et d'évaluation des politiques de l'exécutif. Les parlementaires au sein de ces commissions ont une influence et une maitrise dans certains thèmes qui dépassent de loin celles des parlementaires lambda. Les committees jouent ainsi un rôle important pour influencer les débats parlementaires, les débats au sein de la société civile, ainsi que les discussions sur les différentes propositions de $10 i^{4}$. Par ailleurs, même si cela se réalise de manière moins fréquente au niveau du Parlement européen, les différentes assemblées ont toutes deux la possibilité d'auditionner les membres de leurs exécutifs pour leur poser des questions relatives à leurs politiques via ces commissions parlementaires. Elles ont ainsi la possibilité d'aborder en débat l'ensemble des sujets qui leur semblent importants et d'exprimer leur position sur tous les domaines relevant d'un intérêt à leurs yeux. Ce qui est très important pour la création de l'espace public européen et américain ainsi que pour la légitimation des politiques gouvernementales ${ }^{5}$. Les deux institutions peuvent donc être considérées comme des parlements très puissants de manière générale même si, à l'instar de la majorité des parlements, cette puissance est moindre en matière de politique étrangère.

Il parait également important de noter qu'une coopération interparlementaire entre le Parlement européen et le Congrès américain existe depuis déjà plusieurs années. Les premières

\footnotetext{
${ }^{1}$ Article 1 , section 1 et 8, United States Constitution, signée à Philadelphie le 17 septembre 1787, modifiée le 7 mai 1992.

${ }^{2}$ Article 17, paragraphe 2 du Traité sur l'Union Européenne (TUE), signé à Maastricht le 7 février 1992, modifié le 13 décembre 2007 ; Article 225 et 289 du Traité sur le Fonctionnement de l'Union européenne (TFUE), signé à Rome le 25 mars 1957, modifié le 13 décembre 2007.

${ }^{3}$ BEAUSSIER, A.-L. et BENJABALLAH, S. (2014), « Comparer le Parlement européen et le Congrès américain. Un état des lieux théorique et méthodologique », Revue internationale de politique comparée, vol. $21, \mathrm{n}^{\circ} 3, \mathrm{pp} .9$ 30.

${ }^{4}$ WINZEN, T. (2011), « Technical or Political? An Exploration of the Work of Officials in the Committees of the European Parliament », The Journal of Legislative Studies, vol. 17, n 1, pp. 27-44 ; DAVID, C-P. (éd.) (2015), La politique étrangère des Etats-Unis : fondements, acteurs, formulation, Paris, Presses de Sciences Po, $3^{\text {ème }}$ édition, pp. 414-462.

${ }^{5}$ COSTA, O. (2014), « Que peut le Parlement européen ? », Pouvoirs, vol. 149, n 2, pp. 77-89 ; COURMONT, B. (2001), «Le Congrès et son poids dans la formulation de la politique étrangère américaine », Revue internationale et stratégique, vol. 42, $\mathrm{n}^{\circ} 2$, pp. 87-97.
} 
réunions bilatérales remontent à 1972 et leurs fréquences ont considérablement augmenté depuis deux décennies. Les contacts entre les deux institutions ne sont ainsi pas nouveaux et ils auraient plutôt tendance à être réguliers ${ }^{1}$. Deux éléments peuvent appuyer ce constat, d'une part le Parlement européen dispose d'un bureau de liaison permanent établi à Washington chargé d'entretenir des contacts avec le Congrès américain ${ }^{2}$. Il existe également une structure de dialogue unique entre les deux assemblées à travers laquelle les principaux contacts interparlementaires ont lieu, à savoir le Transatlantic Legislator Dialog (TLD) créé en 1999. Les deux assemblées se rencontrent ainsi en son sein officiellement deux fois par an, mais aussi de manière un peu plus informelle le reste du temps via des groupes de travail communs ou des vidéo-conférences dans le but d'améliorer les échanges, la communication et la compréhension mutuelle ${ }^{3}$.

\section{b. Le Parlement européen et la politique étrangère}

Les compétences du Parlement européen en matière de politique étrangère sont reprises dans le titre 5 du Traité sur le Fonctionnement de l'UE (TFUE). L'article 218 de ce dernier établit ainsi le pouvoir de co-approbation dont dispose l'institution concernant les accords internationaux portant sur des notions clés comme le commerce, l'agriculture et la sécurité intérieure. Il établit également le droit de l'institution à être informée à toutes les étapes de la procédure et de pouvoir saisir la Cour de Justice de l'Union Européenne (CJUE) sur la compatibilité d'un accord international avec les traités de l'UE'.

Parallèlement, la Commission européenne a le monopole pour négocier les accords commerciaux européens sur base d'un mandat préalablement accordé par le Conseil européen. Le rôle de l'assemblée se limite à l'approbation de l'accord négocié précédemment par la Commission. De manière indirecte, le Parlement européen a également le pouvoir d'auditionner les commissaires lorsque ceux-ci interviennent de manière directe ou indirecte sur la politique extérieure. Le haut représentant aux affaires externes a par ailleurs le devoir de consulter et d'informer régulièrement les parlementaires sur son action dans le cadre de la politique européenne de défense et de sécurité commune. Enfin, l'approbation du budget européen, et donc des ressources allouées à la politique extérieure de l'UE, fait-elle aussi partie des compétences du Parlement européen ${ }^{5}$.

Depuis 2009 et la mise en application du traité de Lisbonne, ces différents pouvoirs du Parlement européen ont fait l'objet de nombreuses études et analyses par les chercheurs. En matière de « politique étrangère européenne ${ }^{6}$, plusieurs auteurs ont ainsi affirmé, en se basant sur les compétences reprises dans les traités, que les pouvoirs de cette assemblée se limitent à

\footnotetext{
${ }^{1}$ JANČIĆ, D. (2016), «The Role of the European Parliament and the US Congress in Shaping Transatlantic Relations: TTIP, NSA Surveillance, and CIA Renditions », Journal of Common Market Studies, vol. 54, $\mathrm{n}^{\circ} 4$, pp. 896-912 ; Transatlantic Legislators' Dialogue (2019), «Transatlantic Legislators' Dialogue: Welcome », europa.eu, https://www.europarl.europa.eu/intcoop/tld/default_en.htm.

2 European Parliament (2020), «Bureaux de liaison dans votre pays », europa.eu, http://www.europarl.europa.eu/at-your-service/fr/stay-informed/liaison-offices-in-your-country.

3 Transatlantic Legislators' Dialogue (2019), "Transatlantic Legislators' Dialogue: Welcome », europa.eu, https://www.europarl.europa.eu/intcoop/tld/default_en.htm ; JANČIĆ, D. (2016), op. cit.

4 Article 218 du Traité sur le Fonctionnement de l'Union européenne (TFUE), signé à Rome le 25 Mars 1957, modifié le 13 Décembre 2007. ; Damen, D. (2019), «L’Union européenne et ses partenaires commerciaux », Fiches thématiques l'Union sur européenne, Novembre http://www.europarl.europa.eu/factsheets/fr/sheet/160/l-union-europeenne-et-ses-partenaires-commerciaux.

5 RIPOLL SERVENT, A. (2014), «The role of the European Parliament in international negotiations after Lisbon », Journal of European Public Policy, vol. 21, n 4, pp. 568-586 ; KEUKELEIRE, S., DELREUX, T. (2014), The Foreign Policy of the European Union, Londres, MacMillan Education UK, 2 ème edition, pp. 85-88. ${ }^{6}$ Le terme «politique étrangère » doit être ici nuancé car elle se divise étrangère entre la PESC, qui est restée intégralement intergouvernementale et dont le PE est totalement exclu, et la politique commerciale, compétence exclusive de l'Union où le PE est très impliqué, ce qui diffère avec la politique étrangère d'un État traditionnel.
} 
son pouvoir de codécision en matière du budget de l'Union et à son pouvoir d'approuver ou de rejeter l'adoption des accords internationaux, ce dernier trait s'apparentant à un droit de véto. Son implication dans la politique étrangère de l'UE est donc, selon eux, à priori très faible ${ }^{1}$. Néanmoins, la grande majorité des chercheurs mettent en avant l'extension importante des pouvoirs que le Parlement européen a connu depuis 2009. Pour les auteurs comme Ariadna Ripoll Servent, Karina L. Pasquariello Mariano et Bruno Theodoro Luciano, l'assemblée a réussi à s'imposer progressivement dans la politique étrangère de l'Union et à s'impliquer dans l'ensemble du processus de négociation et de décision en matière d'accord international ${ }^{2}$. Cette évolution a été rendue possible de par la menace que représentait son pouvoir de véto sur les différents accords négociés par la Commission européenne. Les parlementaires ont ainsi rendu essentielles la consultation et l'implication indirecte du Parlement dans les négociations pour la bonne conduite de ces dernières. À cette fin, l'assemblée a montré à plusieurs reprises qu'elle était prête à appliquer son droit de véto final si elle désapprouvait majoritairement un accord pour lequel elle aurait été trop peu consultée ${ }^{3}$. Cela a notamment été le cas pour les négociations $\mathrm{du}$ "SWIFT agreement ${ }^{4}$. Le Parlement a donc évolué pour devenir un acteur définissant en partie l'agenda politique des négociations de l'UE afin d'éviter le paradigme de la pensée unique.

En outre, grâce à une interprétation extensive de ses propres domaines de compétences, le Parlement a réussi à légitimer son droit d'être impliqué dans les négociations internationales dans des domaines plus variés que ce que prévoyaient les traités et a progressivement gagné en influence dans ces derniers. Enfin, grâce à son pouvoir d'audition, de résolution, d'interrogation et dans une moindre mesure grâce à son travail d'expertise et d'analyse sur les politiques et opérations de l'UE réalisé par les commissions parlementaires, le Parlement a réussi à créer un véritable dialogue avec la Commission européenne en matière de politique étrangère et a pu se greffer à l'ensemble des domaines de cette dernière ${ }^{5}$. Même si le degré d'implication varie fortement encore d'un sujet à l'autre et d'un accord à l'autre ${ }^{6}$. Son influence reste par ailleurs beaucoup plus importante en matière de politique commerciale que dans les autres domaines ${ }^{7}$.

Il est admis par les différents auteurs consultés que cette évolution du rôle du pouvoir législatif européen a été rendue possible car il y a eu une réelle volonté de la part des dirigeants européens de donner une légitimité à l'UE et à sa politique extérieure, notamment commerciale, en incluant davantage le Parlement ${ }^{8}$.

\footnotetext{
${ }^{1}$ KEUKELEIRE, S., DELREUX, T., op. cit. ; FROMAGE, D. (2018), « The European Parliament in the postcrisis era: an institution empowered on paper only? », Journal of European Integration, vol. 40, n 3, pp. 281-294. ${ }^{2}$ RIPOLL SERVENT, A., op. cit. ; PASQUARIELLO MARIONO, K. L. et THODORO LUCIONA, B. (2018), «The parliamentarization of EU trade policy: unveiling the European Parliament's involvement in EUMERCOSUR trade negotiations », European Politics and Society, vol. 20, n 5, pp. 591608.

${ }^{3}$ Ibid.

${ }^{4}$ European Parliament (2010), « SWIFT: European Parliament votes down agreement with the US », europa.eu, 11 février 2010, https://www.europarl.europa.eu/sides/getDoc.do?pubRef=-//EP//TEXT+IMPRESS+20100209IPR68674+0+DOC+XML+V0//EN

${ }^{5}$ RIDDERVOLD, M. et ROSÉN, G. (2016), « Trick and treat: how the Commission and the European Parliament exert influence in EU foreign and security policies », Journal of European Integration, vol. 38, ${ }^{\circ}$ 6, pp. 687-702; PASQUARIELLO MARIONO, K. L. et THODORO LUCIONA, B., op. cit.

${ }^{6}$ HUBER, K. (2015), « The European Parliament as an actor in EU border policies: its role, relations with other EU institutions, and impact », European Security, vol. 24, n 3, pp. 420-437; RIDDERVOLD, M. et ROSÉN, G., op. cit.

7 ROSÉN, G. (2017), «The impact of norms on political decision-making: how to account for the European Parliament's empowerment in EU external trade policy », Journal of European Public Policy, vol. 24, $\mathrm{n}^{\circ}$ 10, pp. 1450-1470.

${ }^{8}$ ROSÉN, G., op. cit. ; RIDDERVOLD, M. et ROSÉN, G., op. cit.
} 


\section{c. Le pouvoir du Congrès américain en politique étrangère}

Historiquement et dans une interprétation stricte de la Constitution américaine, le Congrès américain est supposé être l'institution prédominante en matière de politique extérieure du pays. Ainsi, selon l'article 1 section 8 et l'article 2 section 1, 2, 3 de la Constitution américaine, le Congrès a le pouvoir de réglementer le commerce avec les nations étrangères, de déclarer la guerre, de prévoir des sanctions politiques et économiques vis-à-vis des autres nations, etc. Le président par ailleurs est constitutionnellement obligé d'obtenir l'approbation du Sénat pour, entre autres, la ratification des traités internationaux, la nomination d'ambassadeurs et la nomination du secrétaire d'État. Enfin, le chef de l'État est obligé d'informer périodiquement le Congrès de l'évolution de la politique étrangère du pays ${ }^{1}$.

Sur le papier donc, le Congrès américain est très puissant pour l'orientation de la politique extérieure du pays. Cependant dans la pratique, comme l'on va le constater, les choses ont évolué pour donner progressivement un pouvoir prédominant au président et à son administration dans ce domaine.

L'analyse ne s'attardera pas ici sur la répartition des compétences de «hard power » des États-Unis car il est peu pertinent de vouloir les comparer avec les pouvoirs presque inexistants de l'UE en la matière. Il faut néanmoins retenir qu'aujourd'hui la mainmise du président sur ce domaine est presque absolue face à un Congrès disposant d'un pouvoir de contrôle plus que limité dans la pratique ${ }^{2}$.

Dans les matières civiles, les choses ont évolué depuis la ratification de la Constitution américaine. Tout comme le Parlement européen, le Congrès a le pouvoir de se prononcer sur n'importe quel sujet, politique étrangère comprise, mais ces interventions restent de simples déclarations sans effets concrets ${ }^{3}$. Les deux principaux pouvoirs officiels du Congrès se trouvent réellement au niveau d'une partie des traités internationaux et de la validation du budget annuel accordé à l'exécutif.

En matière d'accords internationaux, il existe dans le droit américain trois types de traités pouvant être mis en place aux États-Unis. Le degré d'implication du Congrès dans les négociations variera en fonction que l'on soit dans l'une ou l'autre procédure. Il existe d'une part le traité traditionnel dont le processus de ratification est extrêmement lourd et difficile à faire passer par le Congrès. Il faut ainsi l'accord de 2/3 des représentants au Sénat pour qu'un traité soit ratifié par l'institution. Il existe ensuite les «executive agreements », traités impliquant uniquement l'exécutif et ratifiés par le président dans les domaines de compétence relevant de ses prérogatives en tant que chef d'État. ${ }^{4}$ L'implication du Congrès est nulle dans cette procédure.

Enfin, la dernière stratégie de ratification d'un accord international est le « congressional-executive agreement ». Depuis 1974, le Congrès des États-Unis peut, s'il vote dans ce sens, accorder un mandat à l'exécutif pour une durée limitée permettant la mise en place d'une procédure exceptionnelle de ratification d'accords internationaux portant sur le commerce : la «trade promotion authority » (TPA) ou «fast track procedure ». Selon les modalités de cette dernière, les accords commerciaux sont négociés par le président et ils

\footnotetext{
1 Article 1 section 8 et l'article 2 section 1, 2 et 3, United States Constitution, signée à Philadelphie le 17 septembre 1787, modifiée le 7 mai 1992.

${ }^{2}$ BRICART, V. (2017), « Le Congrès américain et les pouvoirs de guerre : de la théorie à la pratique », CEFIR Working Paper, ${ }^{\circ}$ 3, pp. 1-19; ROEHRIG, T. et WESSEL, L. A. (2011), « Congress and U.S.-North Korean Relations: The Role of the Entrepreneur », Asian Affairs: An American Review, vol. 38, n 2, pp. 89-108.

${ }^{3}$ Article 1 , section 1 et 8 , United States Constitution, signée à Philadelphie le 17 septembre 1787, modifiée le 7 mai 1992.

${ }^{4}$ FORTMANN, M. (dir.) et MARTIN P. (dir.) (2013), Le système politique américain, Montréal, Les Presses de 1'Université de Montréal, $5^{\text {ème }}$ édition, pp. 421-426 ; KANDEL, M. (2016), « Le rôle du Congrès américain dans le processus de décision en politique étrangère : les accords de Dayton (1995) », Critique internationale, vol. 71, $\mathrm{n}^{\circ} 2$, pp. $109-127$.
} 
doivent répondre positivement aux critères et préoccupations définis dans des domaines spécifiques par le Congrès. Une fois l'accord conclu, les parlementaires ont ainsi l'occasion de se prononcer, 60 jours après l'introduction de la demande de ratification et avec un nombre de débats limité, pour décider à la simple majorité dans les deux chambres s'ils souhaitent approuver la ratification de l'accord commercial. La loi obtenue par ce dernier vote sera appelée le «congressional-executive agreement». Aucune demande d'amendement à l'accord commercial conclu par le président avec les autres parties n'est possible durant les débats entourant le vote. L'administration doit en contrepartie avoir respecté l'ensemble des objectifs de négociation définis par le Congrès dans le mandat qu'elle lui a accordé via la "trade promotion authority ». Elle doit par ailleurs accepter la présence de conseillers nommés par les deux chambres dans l'équipe de négociations et respecter les dispositions qui appellent à notifier et à consulter le Congrès aux étapes prévues par ce dernier. Si le Congrès considère que ces règles et objectifs n'ont pas été atteints, il pourra ainsi refuser de ratifier l'accord s'il est soumis à un vote ou forcer le président via des tractations en dehors du cadre législatif à en renégocier certaines parties avec les autres États avant de le soumettre au vote ${ }^{1}$. Techniquement, un mandat n'est pas nécessaire pour que l'exécutif entame ou même conclue des négociations commerciales avec une autre Nation, mais il est néanmoins requis dans les pratiques coutumières et légales américaines que le Congrès soit informé si un début de négociation est envisagé et il est admis que son implication via cette procédure est essentielle. D'une part pour l'expression de la compétence commerciale des deux chambres législatives dans ce domaine, ensuite pour que l'accord obtienne une légitimité démocratique, enfin pour garantir la bonne mise en œuvre de l'accord sur le territoire américain ${ }^{2}$.

En outre, le Congrès peut autoriser le représentant américain au commerce extérieur (USTR) à enquêter sur les pratiques commerciales déloyales d'une autre nation et à y répondre ${ }^{3}$.

Le Congrès américain reste par ailleurs très influent en matière de politique étrangère grâce à son vaste pouvoir d'enquête, d'audition et de supervision parlementaires conduit par ses commissions ${ }^{4}$. Ce pouvoir d'enquête et d'investigation des parlementaires américains peut parfois les conduire à prendre des initiatives diplomatiques exceptionnelles dans le but d'obtenir une meilleure compréhension des thèmes qu'ils étudient ainsi que pour établir des contacts à des degrés variés avec les différents acteurs impliqués, notamment en se rendant directement sur le terrain ${ }^{5}$.

Enfin, le Congrès américain dispose d'un grand levier pour influencer la politique extérieure notamment grâce à son pouvoir de validation du budget alloué à la politique étrangère du pays. Il dispose ainsi du pouvoir de refuser de financer ou de poursuivre le financement d'un projet ou d'une initiative si cette dernière ne lui convient pas. En relations internationales, ce pouvoir est d'autant plus important que certains traités internationaux nécessitent parfois des fonds importants, de même que la coopération technique et législative du Congrès pour leurs bonnes mises en application dans la législation américaine ${ }^{6}$.

\footnotetext{
${ }^{1}$ DEL MONTE, M. et PUCCIO, L. (2016). Rôle du Congrès des États-Unis dans les accords commerciaux, La procédure accélérée «Fast-Track procedure » (PE 577.999), Bruxelles, European Parliament Research Service ; JANČIĆ, D. (2016), op. cit. ; JANČIĆ, D. (2017), « TTIP and legislative-executive relations in EU trade policy », West European Politics, vol. 40, n 1, pp. 202-221.

${ }^{2}$ FERGUSSO, I.-F. (2015), « Trade Promotion Authority (TPA) and the Role of Congress in Trade Policy (RL33743) », Washington, Congressional Research Service; JANČIĆ, D. (2015), « Transatlantic Regulatory Interdependence, Law and Governance: The Evolving Roles of the EU and US Legislatures », Cambridge Yearbook of European Legal Studies, vol. 17, pp. 334-359.

${ }^{3}$ FERGUSSO, I.-F., op. cit.

${ }^{4}$ COURMONT, B., op. cit. ; JANČIĆ, D. (2015), op. cit.

${ }^{5}$ ROEHRIG, T. et WESSEL, L. A., op. cit. ; BERGBOWER, M. L. et SCHMITT, C. (2015), « Congressional Activism in the Foreign Policy Arena: A Case Study of the 1994 Rwandan Genocide », Democracy and Security, vol. 11, n 4, pp. 353-372 ; KANDEL, M., op. cit.

${ }^{6}$ COURMONT, B., op. cit.
} 


\section{2. Études des accords commerciaux}

Après avoir étudié la théorie entourant les compétences parlementaires en politique extérieure, il est temps d'analyser une série de cas pratiques dans l'optique de confirmer ou d'infirmer ces théories contingentes. Il faut cependant préciser que cette analyse se limitera à l'étude du respect et de l'application des compétences principales du Congrès et du Parlement européen en matière commerciale. Les éléments secondaires, mais importants, comme le rôle des partis politiques, l'influence des personnalités dirigeantes parlementaires, l'impact du pouvoir budgétaire et enfin l'influence des parlements nationaux ou des entités fédérées sur les accords commerciaux ne seront pas traités ici dans l'optique de ne pas perdre le fil conducteur de notre analyse.

\subsection{Le Parlement européen}

\section{a. TTIP}

Dès les premières phases de dialogue entre négociateurs, le Parlement européen a manifesté son souhait de s'impliquer dans le contenu des négociations du TTIP entre les ÉtatsUnis et l'UE. Ainsi durant l'année 2013, soit la première année des pourparlers, l'assemblée a rappelé rapidement à la Commission le rôle que les parlementaires devraient jouer et elle a également défini les objectifs, les priorités et les problématiques importantes auxquels il faudrait répondre afin que les futures négociations soient menées à bien. Cela s'est exprimé d'une part à travers sa résolution du 23 mai $2013^{1}$, mais également via ses différentes compétences d'information et d'enquête. Dans cette optique, l'audition de membres de la Commission européenne par 15 des 20 commissions parlementaires ou groupes de recherche différents a été cruciale pour maintenir au-devant de la scène ses demandes et pour faire évoluer les débats entourant les négociations ${ }^{2}$. Dans ces dernières, on peut y retrouver de nombreuses questions relatives à la transparence, ou plutôt au manque de transparence, des négociations entre la Commission et les négociateurs américains. Par ailleurs, l'assemblée a manifesté de sérieuses préoccupations concernant la nature du système de « règlement des différends entre un investisseur et un État » prévu par le traité. Les éléments connus de l'époque mettaient ainsi en avant une pratique juridique favorisant davantage les entreprises et étant contraire aux pratiques de règlements des différends en Europe. Elle a ainsi fait de la modification de ce système une condition sine qua non à sa ratification du traité ${ }^{3}$. Le Parlement a obtenu par ailleurs que soient mis en place des « gardes fous » portant sur certaines matières sensibles pour les consommateurs européens ${ }^{4}$.

\footnotetext{
${ }^{1}$ European Parliament, «EU trade and investment agreement negotiations with the US », Resolution, P7_TA (2013)0227, 23 May 2013.

${ }^{2}$ ROEDERER-RYNNING, C. (2017), « Parliamentary assertion and deep integration: the European parliament in the CETA and TTIP negotiations », Cambridge Review of International Affairs, vol. 30, n 5-6, pp. 507-526 ; European Parliament, «TTIP: Challenges and Opportunities for the Internal Market », Joint Public Hearing Committee on the Internal Market and Consumer Protection Committee on International Trade, http://www.europarl.europa.eu, 24 February 2015 ; Committees (2020), « Hearings », European Parliament Committees, https://www.europarl.europa.eu/committees/en/indexsearch.html?term=9\&query=TTIP ; European Parliament, «TTIP: What's in it for Europeans?», Public Hearing Committee on International Trade, DV $1053409 E N . d o c, 18$ March 2015.

${ }^{3}$ PUCCIO, L. (2016). Négociations UE/États-Unis sur le PTCI, Vue d'ensemble des questions en suspens (PE 586.606), Bruxelles, European Parliament Research Service ; JANČIĆ, D. (2016), op. cit.

${ }^{4}$ Ibid; European Parliament, «European Parliament's Recommendations to the European Commission on the Negotiations for the Transatlantic Trade and Investment Partnership (TTIP) », Resolution, P8_TA(2015)0252, 8 July 2015.
} 
Dans cette situation, la participation du Parlement européen est clairement visible et l'on constate également en regardant l'évolution du discours et des publications des représentants de la Commission européenne que les préoccupations de l'assemblée ont été largement prises en considération par ses négociateurs ${ }^{1}$. D'une part, à partir de 2015, notamment après la résolution du Parlement européen du 8 juillet dans laquelle il a exposé ses 69 préoccupations, recommandations et objectifs concernant le traité ${ }^{2}$, le contenu des négociations a été régulièrement publié et transmis aux parlementaires ${ }^{3}$. La publication de ces documents a rendu possible son contrôle et son évaluation par les parlementaires, ce qui leur a permis d'émettre des réserves sur différents points. Ensuite, face aux importantes critiques, le système de règlements des différends a été abandonné dans le traité et remplacé par un «tribunal de règlement des différends en matière d'investissement». Par ailleurs, une partie des revendications du Parlement ont été par la suite reprises dans la position officielle de la Commission pour les négociations ${ }^{4}$.

Pour bien comprendre ce qui a poussé et permis au Parlement européen de s'impliquer autant dans le processus de négociations, il faut prendre en compte l'extrême impopularité et inquiétude qui étaient ressentie par la société civile vis-à-vis de l'accord du TTIP ${ }^{5}$, notamment après la polémique entourant le système de règlement des différends en matière d'investissement. Ainsi fin 2014 la Commission Juncker, au fil de plusieurs sondages d'opinion ou de consultations populaires confirmant l'impopularité du TTIP et de son système de règlement des différends ${ }^{6}$, a fini par céder au besoin de transparence réclamé par le Parlement pour les négociations afin de lutter contre le caractère hautement impopulaire de l'accord. Pour Alina Alexoaei et Valentin Cojanu ce n'est donc pas la demande d'implication accrue du Parlement aux négociations commerciales qui poussa la Commission à réagir, mais bien la volonté de la nouvelle Commission Juncker de légitimer l'accord auprès de la société civile afin de faire face à la prolifération des discours de plus en plus nombreux de certains groupes politiques, au niveau européen ou au niveau des États, déclarant qu'ils n'allaient pas soutenir pas l'accord sans transparence au préalable. Via sa mauvaise campagne de communication autour du TTIP, la Commission a ainsi contribué à rendre nécessaire un contrôle législatif de

\footnotetext{
${ }^{1}$ European Commission (2015), «Dans un souci de transparence, la Commission européenne publie des textes juridiques concernant le TTIP », europa.eu, 7 janvier 2015, https://europa.eu/rapid/press-release IP-152980 fr.htm ; Malmström, C. (2014), «The Trade Commissioner and Parliament: A Vital Relationship », europa.eu, 3 décembre 2014, https://europa.eu/rapid/press-release_SPEECH-14-2348_en.htm ; European Commission (2015), «Présentation ce jour d'un rapport sur la consultation concernant la protection des investissements dans les négociations commerciales UE-Etats-Unis », europa.eu, 13 janvier 2015, https://europa.eu/rapid/press-release_IP-15-3201_fr.htm.

2 European Parliament, «European Parliament's Recommendations to the European Commission on the Negotiations for the Transatlantic Trade and Investment Partnership (TTIP) », Resolution, P8_TA(2015)0252, 8 July 2015.

3 European Commission (2016), «EU negotiating texts in TTIP », europa.eu, 14 juillet 2016, http://trade.ec.europa.eu/doclib/press/index.cfm?id=1230.

4 PUCCIO, L., op. cit.; European Commission (2016), «EU negotiating texts in TTIP», europa.eu, 14 juillet 2016, http://trade.ec.europa.eu/doclib/press/index.cfm?id=1230.

5 DUCOURTIEUX, C. (2016), «La galaxie hétéroclite des anti-TTIP », Lemonde, 16 septembre ; DUCOURTIEUX, C. (2016), «Les autorités peinent à convaincre les opinions publiques », Lemonde, 30 avril.

${ }^{6}$ European Commission. (2015). Online public consultation on investment protection and investor-to-state dispute settlement (ISDS) in the Transatlantic Trade and Investment Partnership Agreement(TTIP) (13.1.2015 SWD (2015) 3 final), Bruxelles, http://trade.ec.europa.eu/doclib/docs/2015/january/tradoc 153044.pdf ; European Commission (2014), « Online public consultation on investment protection and investor-to-state dispute settlement (ISDS) in the Transatlantic Trade and Investment Partnership Agreement (TTIP) », europa.eu, juillet 2014, http://trade.ec.europa.eu/consultations/index.cfm?consul_id=179 ; European Commission (2015), «Présentation ce jour d'un rapport sur la consultation concernant la protection des investissements dans les négociations commerciales UE-Etats-Unis », europa.eu, 13 janvier 2015, https://europa.eu/rapid/press-release IP-153201_fr.htm.
} 
l'accord ${ }^{1}$. Pour reprendre les propos de Christilla Roederer-Rynning « en exploitant le pouvoir de la mobilisation sociale, le Parlement européen n'a pas seulement concrétisé de manière concrète ses droits à une information complète découlant d'un traité. Il a également contribué, par son activisme juridique et constitutionnel, à ouvrir le processus décisionnel des négociations commerciales internationales et a obligé la Commission et le Conseil à accepter un degré de transparence accru $»^{2}$.

\section{b. CETA}

Les négociations pour le Comprehensive Economic and Trade Agreement (CETA) entre l'UE et le Canada ont débuté en mai 2009, quelques mois avant que les différentes réformes du traité de Lisbonne accordant un pouvoir de codécision au Parlement européen aient été entièrement mises en place. Ainsi, ce n'est que fin de la même année que le Parlement a entrepris d'exercer ses compétences en matière de contrôle et de supervision dans cette négociation commerciale. Néanmoins, du début des dialogues en 2009 jusqu'à la fin de ces derniers en 2014, le nombre d'interventions et de rapports parlementaires sur le CETA est resté relativement peu élevé si on le compare avec celui du TTIP. Par ailleurs, sur cette même période seule une commission parlementaire sur les 20 existantes s'est impliquée dans le contrôle du processus $^{3}$. Pour Chritilla Roederer-Rynning, si les interventions du Parlement européen sur les négociations ont été réelles, ces dernières étaient beaucoup moins communiquées au public que pour le TTIP, notamment en raison d'une certaine ignorance ou d'un désintérêt médiatique pour l'accord. Le Parlement lui-même avait souhaité se concentrer davantage sur le contrôle de l'action du Conseil et de la Commission plutôt que sur sa communication avec les citoyens européens et la société civile. Enfin, il a existé à l'époque parmi les parlementaires un sentiment partagé que le Canada était suffisamment proche culturellement et économiquement des pratiques européennes pour qu'il ne soit pas nécessaire de polémiquer sur le sujet. Il y a donc eu dans un premier temps un consensus d'adhésion à l'idée d'un partenariat économique avec ce pays ${ }^{4}$.

Une première confrontation entre le Parlement européen et la Commission a néanmoins débuté en 2011 notamment via l'apparition des premiers dossiers polémiques. Ainsi, les parlementaires ont progressivement commencé à s'intéresser à certaines informations communiquées sur le contenu du traité qui les ont conduites à faire une première série de réclamations et recommandations, notamment via la résolution du Parlement du 8 juin $2011 .^{5}$ C'est principalement dans cette dernière que l'assemblée a fixé ses préoccupations et guidelines majeures concernant l'accord avec le Canada. On y retrouve des préoccupations concernant la protection de la qualité des produits européens, la protection de l'environnement, mais également sur le respect de la nouvelle répartition des compétences accordant un plus grand rôle du Parlement européen dans les négociations. Il a ainsi été rappelé à l'exécutif son obligation de tenir les parlementaires informés au maximum sur l'évolution des négociations et, surtout, il y a été enjoint à la Commission d'attendre d'avoir reçu l'avis émis par le Parlement

\footnotetext{
1 ALEXOAEI, A. et COJANU, V. (2017), « Negotiating the Transatlantic deal: focus on the EU's domestic constraints », CES Working Papers, Centre for European Studies, Alexandru Ioan Cuza University, vol. 9, $n^{\circ} 3$, pp. 233-254.

${ }^{2}$ ROEDERER-RYNNING, C., op. cit.

Ibid.; European Parliament (2019), «European Parliament Committees », europa.eu, https://www.europarl.europa.eu/committees/en/parliamentary-committees.html.

${ }^{4}$ ROEDERER-RYNNING, C., op. cit.

5 European Parliament, «European Parliament resolution of 8 June 2011 on EU-Canada trade relations », Resolution, P7_TA(2011)0257, 8 June 2011.
} 
européen à chaque clôture de volet de négociations avant d'entamer le volet suivant. Règle que la Commission ne respectait pas avant la publication de cette note ${ }^{1}$.

Le point de friction le plus important a probablement été celui portant sur le volet « investissement» des négociations. L'un des impacts majeurs de l'implication du Parlement dans les négociations a ainsi été l'obtention en 2014-2015 la mise en place du Système Juridictionnel des Investissements (SJI) visant à régler les différends entre les États et les investisseurs et remplaçant le système de règlement des différends proposé par les négociateurs. Ce dernier ayant été très polémique parmi les parlementaires et parmi la société civile européenne car considéré comme trop intrusif, la polémique a par ailleurs porté sur le même sujet lors des négociations du TTIP ${ }^{2}$. Si ce nouveau système est resté lui aussi très polémique, il n'en reste pas moins que les négociateurs ont pris au sérieux l'avis négatif du Parlement européen sur ce sujet lorsque ce dernier a affirmé que l'accord ne serait pas adopté tant que le précédent système serait présent dans le traité. Par ailleurs, les parlementaires ont obtenu en 2015 la publication officielle du descriptif du mandat accordé par le Conseil à la Commission pour négocier ${ }^{3}$.

Il est intéressant de noter ici que les concessions majeures obtenues par le Parlement l'ont été après la clôture des négociations en 2014. Deux points peuvent expliquer cette réaction assez tardive du Parlement, de même que les concessions obtenues. D'une part, à la clôture des négociations du CETA en 2014, le public et les médias ont pris conscience de l'existence de ce traité et de nombreux parallèles ont été faits avec le TTIP 4 , contribuant à rendre l'accord d'emblée polémique. D'autre part, après la conclusion des négociations, le manque de transparence et de communication de la Commission a rapidement été critiqué et utilisé pour expliquer l'absence de suivi de l'évolution des négociations par le public européen ${ }^{5}$. Dès lors, face à ces polémiques, le Parlement a su une fois encore rebondir sur ces dernières afin d'émettre de nouvelles revendications ne pouvant être ignorées par la Commission.

Au terme des négociations, l'opinion du Parlement européen sur son implication dans le processus de négociation et sur la transparence de ces dernières s'est voulue globalement positive. Dans un rapport émis en 2017, il a ainsi constaté avoir largement pu exprimer ses idées et remarques dans la deuxième partie des négociations et il y a reconnu les efforts réalisés par la Commission en matière de transparence et pour tenir compte de l'avis des parlementaires alors même que l'accord avait déjà été conclu' 6 .

Avant sa ratification par le Parlement, plusieurs commissions parlementaires ont finalement pu s'exprimer sur le contenu du traité. Si la grande majorité de celles-ci ont approuvé la ratification du traité grâce aux mesures de protection prises par les négociateurs dans les secteurs sensibles, la Commission parlementaire de l'emploi et des affaires sociales du Parlement européen, elle, s'y est opposé ${ }^{7}$. Enfin, une délégation de la commission parlementaire chargée du commerce extérieur s'est rendue en mars 2016 à Ottawa dans l'optique de dialoguer

\footnotetext{
${ }^{1}$ SCHÖLLMANN, W. (2017), Briefing International Agreements in Progress: Comprehensive Economic and Trade Agreement (CETA) with Canada (PE 595.895), Bruxelles, European Parliament Research Service ; ROEDERER-RYNNING, C., op. cit.

${ }^{2}$ Ibid.

${ }^{3}$ European Parliament. (2019), EU-Canada comprehensive economic and trade agreement (CETA), (Legislative Train $\mathrm{n}^{\circ} 07.2019$ 6A), Bruxelles, https://www.europarl.europa.eu/legislative-train/api/stages/report/072019/theme/a-balanced-and-progressive-trade-policy-to-harness-globalisation/file/ceta.

${ }^{4}$ STEHRER, R. et STÖLLINGER, R. (2016), « CETA and TTIP: same-same but different? », Vienna Institute for International Economic Studies, 6 septembre ; ALEXOAEI, A. et COJANU, V., op. cit., pp. 233-254.

${ }^{5}$ DUCOURTIEUX, C. (2018), «Europe : Bruxelles contrainte à la transparence sous la pression des opinions publiques », Lemonde, 19 mai.

${ }^{6}$ SCHÖLLMANN, W., op. cit.

${ }^{7}$ VINCENTI, D. (2016), « Parliament committee gives CETA thumbs down », euractiv, 9 décembre.
} 
avec les différents groupes et représentants de la société canadienne ${ }^{1}$. De plus, le Parlement européen et le Parlement canadien ont annuellement effectué des réunions interparlementaires. Il ne s'agissait cependant pas de négociations, mais d'échanges visant à améliorer la communication et les compréhensions réciproques entre les deux institutions. Le CETA, bien que peu présent dans les discutions, y a été débattu quant à son image désastreuse dans l'opinion publique et quant aux dernières évolutions dans son contenu ${ }^{2}$. Aucune forme de négociations ou d'impact concret dans le contenu de l'accord ne peut donc en être retirée de ces différents échanges interparlementaires. Au final le traité a été approuvé par le Parlement européen en février $2017^{3}$.

On peut finalement retenir que si elles ne l'étaient pas au début, les négociations du TTIP et du CETA sont devenues par la suite extrêmement liées pour plusieurs raisons. D'une part, leurs négociations respectives se sont déroulées soit de manière concomitante, soit de manière successive. Par ailleurs, le contenu des échanges et des dossiers négociés a été similaire sur de nombreux points et caractéristiques dans les deux traités. Enfin, les polémiques les entourant ont été également semblables, notamment au niveau du système de règlement des différends et du débat sur la transparence. De ce fait, les concessions obtenues par le Parlement pour l'un des dossiers ont été transposées également dans l'autre. En contrepartie, les polémiques associées à l'un des accords en cours de négociation se sont vues également transvasées aux négociations pour l'autre ${ }^{4}$. On peut retenir au final que le point de divergence entre les deux processus fut le début de la période d'implication parlementaire soutenue. Si pour le CETA elle a été tardive, elle a par contre été très intense dès le début des échanges pour le TTIP.

\subsection{Le Congrès américain}

\section{a. TTIP}

Le Congrès américain a de prime abord exprimé un avis favorable sur les négociations entre les États-Unis et l'UE dès 2013. Via une résolution parlementaire, il a ainsi apporté son appui à l'initiative présidentielle et il a par ailleurs recommandé que ce dernier maintienne le Congrès informé sur l'évolution des négociations ${ }^{5}$. Cependant, la véritable mobilisation et implication concrète du Congrès américain dans les négociations sur le TTIP a eu lieu d'une manière différente et plus tardive que pour le Parlement européen. Il a en effet fallu que soit d'abord négociée entre les parlementaires et le président la mise en place de la procédure de

\footnotetext{
${ }^{1}$ Committee on International Trade. (2016). Mission Report: Following the ad-hoc delegation to Canada (Ottawa and Montreal) from 21 to 23 March 2016 ( CR\1089805EN.doc PE578.851v01-00), Bruxelles, European Parliament, https://www.europarl.europa.eu/cmsdata/102098/Canada_Mission_Report.pdf.

2 European Parliament Delegation for Relations with Canada (2019), «Welcome from the former Chair », europa.eu, https://www.europarl.europa.eu/delegations/en/d-ca/home; European Parliament Delegation for Relations with Canada. (2014), Delegation for Relations with Canada 36th EP-Canada Inter-Parliamentary Meeting Brussels 13 November 2014 (Mission Report of 2014), Bruxelles, European Parliament, https://www.europarl.europa.eu/cmsdata/124161/Report\%2036th\%20EP-Canada\%20IPM.pdf ; European Parliament Delegation for Relations with Canada. (2017), Delegation for Relations with Canada 37th EU-Canada Inter-Parliamentary Meeting Ottawa, Quebec and Montreal 16-20 May 2016 (Mission Report of 2016), Bruxelles, European Parliament, https://www.europarl.europa.eu/cmsdata/124140/Mission\%20report.pdf.

${ }^{3}$ European Parliament (2017), « CETA: MEPs back EU-Canada trade agreement », europa.eu, 2 février 2017, http://www.europarl.europa.eu/news/en/press-room/20170209IPR61728/ceta-meps-back-eu-canada-tradeagreement.

${ }^{4}$ ROEDERER-RYNNING, C., op. cit. ; ALEXOAEI, A. et COJANU, V., op. cit.

${ }^{5}$ US House of Representatives, « Expressing the sense of the House of Representatives that the United States and the European Union should pursue a Transatlantic Trade and Investment Partnership », Resolution, H.Res.76 $\left(113^{\text {th }}\right), 15$ February 2013.
} 
ratification simplifiée du traité. Grâce à celle-ci, le Congrès a pu s'impliquer officiellement dans l'analyse et l'évaluation du contenu des négociations dès que le mandat définissant ses objectifs, priorités, attentes et préoccupations concernant les négociations commerciales a été adopté. Néanmoins, cette procédure n'a été mise en place qu'en 2015, soit deux ans après le début des négociations, ces dernières ayant déjà bien avancé ${ }^{1}$. Néanmoins, des séances de briefing sur l'évolution des discussions avaient néanmoins été organisées épisodiquement dès 2013 par les négociateurs afin de briefer les parlementaires américains ${ }^{2}$.

Dès 2015, à travers des auditions parlementaires initiées par les différentes commissions internes des deux chambres, les parlementaires ont pu soumettre leurs préoccupations et interrogations aux négociateurs et experts en matière de commerce ${ }^{3}$. En autorisant la mise en place de la "trade promotion authority ", le Congrès a ainsi pu fixer de manière officielle les critères que l'exécutif se devait de respecter pour que l'accord soit adopté, lui donnant ainsi une influence indirecte, mais concrète sur le contenu de l'accord étant négocié.

Néanmoins, le mandat qui a été accordé en juin 2015 au président pour négocier des accords commerciaux n'était pas spécifique au TTIP, mais avait un caractère général destiné à l'ensemble des accords commerciaux négociés par le président sur une période comprise entre 2015 et le $1^{\text {er }}$ juillet 2018. Le Congrès a dès lors renoncé comme prévu à toute possibilité d'amender lui-même directement l'accord une fois celui-ci conclu. Il a en plus de cela cadré sa propre marge de manœuvre car il lui était impossible d'apporter, en plus des préoccupations développées dans la "trade promotion authority", d'éventuels nouveaux volets de préoccupations spécifiques au TTIP dans les négociations en cours ou après la conclusion éventuelle de celles-ci comme ce fut le cas du Parlement européen pour le TTIP et le CETA ${ }^{4}$.

Au-delà d'une définition de ses priorités, cette procédure lui a également permis d'obtenir un cadre légal faisant valoir son droit à être informé dans la suite de la procédure de négociation. Un représentant officiel chargé de faire respecter cette transparence a ainsi été nommé, de même que deux conseillers spéciaux représentants pour l'un la Chambre des représentants et pour l'autre le Sénat. Ces derniers étant affectés auprès du bureau du représentant américain au commerce extérieur (USTR) chargé des négociations ${ }^{5}$. De manière générale, la pratique des négociateurs américains lors de négociations commerciales est de garder la stratégie et la feuille de route des pourparlers secrètes afin de faciliter la tâche des diplomates. Dans cette optique, l'accès aux documents de négociation du TTIP a ainsi été strictement limité ${ }^{6}$. Néanmoins, de par la nature de la «trade promotion authority », il est avéré que le bureau du représentant au commerce américain a transmis en partie ces informations aux parlementaires et qu'il a fait preuve de plus de transparence que par le passé sur le contenu des négociations ${ }^{7}$. Cependant, pour certains parlementaires cette transparence a été très imparfaite

\footnotetext{
${ }^{1}$ US Congress, « Bipartisan Congressional Trade Priorities and Accountability Act of 2015 », Public Law, 114$26\left(114^{\text {th }}\right)$, Congress, 29 June 2015.

${ }^{2}$ Giovenco, A. (2013), « Chief U.S. and EU Negotiators Brief Congressional Staff on the First Round of TTIP Negotiations », Tradewinds, the official blog of the United States Trade Representative, juillet 2013, https://ustr.gov/about-us/policy-offices/press-office/blog/2013/july/negotiators-brief-hill-ttip.

${ }^{3}$ US House of Representatives, «U.S. Trade Policy Agenda », Hearings Before the Committee of Ways and Means, 114-FC02 $\left(114^{\text {th }}\right)$, 27 January 2015 ; United Sates Senate (2020), « Hearings \& Meetings », senate.gov, https://www.senate.gov/committees/hearings_meetings.htm; US House of Representatives Ways and Means' committee (2015), «Hearing on Trade», Way \& Means Committee, 22 Avril 2015, https://waysandmeans.house.gov/legislation/hearings/hearing-trade ; US House of Representatives, « Expanding American Trade with Accountability and Transparency », Hearings Before the Committee of Ways and Means, 114-FC04 (114 $\left.{ }^{\text {th }}\right)$, Congress, 22 April 2015.

${ }^{4}$ PUCCIO, L., op. cit.

${ }^{5}$ Le représentant américain au commerce est membre de l'exécutif américain.

${ }^{6}$ DAVIES BOREN, Z. (2015), « TTIP controversy: Secret trade deal can only be read in secure 'reading room' in Brussels », TheIndependent, 4 août.

${ }^{7}$ JANČIĆ, D. (2015), op. cit.
} 
et l'on a ainsi assisté à plusieurs polémiques concernant les documents transmis. Ces derniers étant critiqués pour leur contenu trop général ou approximatif. Le manque de transparence de l'exécutif a dès lors été très contesté dans le chef des parlementaires américains ${ }^{1}$.

Malgré tout, on a pu constater une certaine volonté de la part du Congrès à voir un accord aboutir et à poursuivre leur travail avec le représentant américain au commerce ${ }^{2}$.

Parallèlement, une coopération renforcée avec les parlementaires européens a également été encouragée par les représentants américains, notamment à travers le TLD et via plusieurs visites réciproques de délégations parlementaires au sein des assemblées et comités respectifs des deux groupes de négociateurs. Néanmoins, il apparait que si cette institution a permis l'échange d'informations et de point de vue entre parlementaires, il y a eu un manque important d'impact de la coopération interparlementaire sur les négociations ${ }^{3}$.

Les négociations du TTIP ont été mises en pause en 2017 après la prise en charge du nouvel exécutif américain se voulant beaucoup moins favorable aux traités de libre-échange. Il est intéressant de noter que les négociations n'ont pas été officiellement abandonnées, mais bien suspendues pour une durée indéterminée. Il semble cependant peu probable de les voir repartir sur base de ce qui avait déjà été négocié 4 .

\section{b. TPP}

Concernant le TPP, le contenu des négociations des États-Unis avec les pays de la région d'Asie-Pacifique et d'Amérique a pendant très longtemps été gardé secret par l'exécutif américain, ce qui a entrainé nombre de critiques tant dans le chef des parlementaires américains que dans le chef des assemblées des différentes Nations associées aux discussions ${ }^{5}$. Ainsi, les

\footnotetext{
${ }^{1}$ ILIAS AKHTAR, S. et JONES, V. (2014). Transatlantic Trade and Investment Partnership (T-TIP) Negotiations (R43387), Washington, Congressional Research Service, https://fas.org/sgp/crs/row/R43387.pdf.

${ }^{2}$ United States Senate Committee on Finance (2016), « Hatch, Brady Send Letter to USTR as TTIP Negotiations with EU Resume », US Senate Committee on Finance, 3 octobre 2016, https://www.finance.senate.gov/chairmans-news/hatch-brady-send-letter-to-ustr-as-ttip-negotiations-with-euresume ; JANČIĆ, D. (2015), op. cit.

${ }^{3}$ European Parliament Liaison Office in Washington DC (2013, April 11), « Transatlantic Trade and Investment Partnership: MEPs gauge 'increasingly positive mood' in Washington, outline key EU interests », europa.eu, 11 April 2013,

www.europarl.europa.eu/us/en/news events/news/news_2013/news_apr_2013/ttip_eu_interests.html ; European Parliament (2014), «Politics \& Policies - April $2014 », \quad$ europa.eu, 10 Avril 2014, https://www.europarl.europa.eu/unitedstates/en/eplo-news/politics-policies-april-2014; $\quad$ ARC (2014), «Transatlantic Dialogue between legislators on TTIP in European Parliament», Arc 2020, 21 juillet 2014, www.arc2020.eu/front/2014/07/transatlantic-dialogue-between-legislators-on-ttip-in-the-european-parliament ; European Parliament \& US House of Representatives. (2013), 74TH Inter-parliamentary meeting: Transatlantic Legislators' Dialogue Statement of 2013), Bruxelles, https://www.europarl.europa.eu/cmsdata/122911/TLD\%20June\%202013.pdf (consulté en août 2019) ; European Parliament \& US House of Representatives. (2014). 75TH Inter-parliamentary meeting: Transatlantic Legislators'

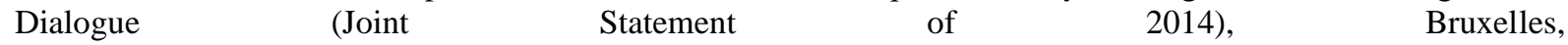
https://www.europarl.europa.eu/cmsdata/122912/TLD\%20March\%202014.pdf (consulté en août 2019); European Parliament \& US House of Representatives. (2015). 77TH Inter-parliamentary meeting: Transatlantic Legislators' Dialogue (2nd Joint Statement of 2015), Bruxelles, https://www.europarl.europa.eu/cmsdata/122148/Joint_Statement_EU-US_IPM 4-5_Nov_2015.pdf (consulté en août 2019).

${ }^{4}$ LAROCCO, L. A. (2017), « Wilbur Ross says he's 'open to resuming' talks on mega-trade deal with Europe », CNBC, 30 mai.

${ }^{5}$ NEW, W. (2012), « US Congress Members Demand Access To TPP; ACTA Criticised In Australia », Intellectual Property Watch, 27 juillet; ERMERT, M. (2015), « TPP Heads Into Ratification Game », Intellectual Property Watch, 6 octobre.
} 
négociations impliquant les États-Unis ont débuté en 2008 et, mis à part quelques fuites d'informations ${ }^{1}$, l'entièreté du contenu de ces dernières n'a été rendu publique qu'en novembre 2015, soit une fois que les négociations se sont terminées ${ }^{2}$. Ce manque de transparence a créé de nombreuses inquiétudes sur des sujets divers et variés dans le chef de la société civile américaine ainsi que dans le chef de plusieurs parlementaires américains relayant cette dernière. Inquiétudes par ailleurs similaires à celles qui ont été émises contre le TTIP à la même époque, mais avec cependant plus de virulence de la part des opposants à l'accord et avec davantage d'impact sur l'opinion publique'.

Si le président Barack Obama a remporté plusieurs victoires sur ce dossier, notamment lorsque le Congrès a adopté un nouveau «trade act» en juin 2015 initiant ainsi la procédure de ratification simplifiée, ainsi qu'une seconde victoire en novembre de la même année en ayant réussi à achever les négociations du TPP, il n'a jamais obtenu de vote du Congrès sur le document final de cet accord commercial ${ }^{4}$. Plusieurs raisons sont avancées pour donner une explication à cet échec.

D'une part, les négociations ont été maintenues secrètes pendant une très longue période et leur contenu n'a été complètement divulgué qu'en novembre 2015. Au cours des années précédentes, le Congrès a tenté en vain à plusieurs reprises d'obtenir des détails sur celles-ci. Ainsi, des auditions parlementaires ont eu lieu et de nombreux membres du Congrès ont exprimés leurs points de vue et préoccupations sur les négociations via des lettres publiques ainsi qu'au travers de consultations directes avec les membres de l'administration et de groupes d'influence ${ }^{5}$. Certaines séances de consultation de l'accord ont également été organisées avant la mise en place de la «trade promotion autority », néanmoins le nombre de parlementaires y ayant eu accès a été extrêmement limité et les conditions de consultations ont été très strictes afin de maintenir le secret sur le contenu de $1^{1}$ accord ${ }^{6}$. À terme, les différentes interventions et actions au Congrès ont eu peu d'impact sur l'attitude de l'exécutif, le manque de transparence des négociations s'est maintenu durant tout le processus diplomatique, augmentant et confortant le nombre d'opposants à cet accord tant dans le camp des législateurs démocrates, que dans celui des législateurs républicains ${ }^{7}$.

Une fois que les négociations ont été terminées et que l'accord commercial a été signé en février 2016 par le président, la suite logique aurait été la mise en place au Congrès de discussions sur la transposition du contenu du traité dans le droit américain, puis sur sa ratification par les deux chambres législatives ${ }^{8}$. Néanmoins, les élections présidentielles américaines sont venues chambouler ce processus. En effet, le mandat de Barack Obama arrivant à terme fin 2016, le président n'a disposé que d'une année pour faire adopter le TPP avant de voir son mandat se terminer. Sachant que l'ensemble des candidats à la présidentielle

1 THE GUARDIAN (2013), «WikiLeaks publishes secret draft chapter of Trans-Pacific Partnership », TheGuardian, 13 ovembre ; Wikileaks (2014), « Updated Secret Trans-Pacific Partnership Agreement (TPP) - IP Chapter (second publication) », wikileaks, 16 octobre 2014, https://wikileaks.org/tpp-ip2/.

${ }^{2}$ BINDER, K. (2017). Briefing: From TPP to new trade arrangements in the Asia-Pacific region (PE 603.953), Bruxelles, European Parliament Research Service.

${ }^{3}$ WILLIAMS, B.-R. et FERGUSSON, I.-F. (2018). TPP: Overview and Current Status. (IF10000 Version n ${ }^{\circ} 28$ ), Washington, Congressional Research Service.

${ }^{4}$ US Congress, « Bipartisan Congressional Trade Priorities and Accountability Act of 2015 », Public Law, 114$26\left(114^{\text {th }}\right)$, Congress, 29 June 2015.

${ }^{5}$ CALMES, J. (2015), «Trans-Pacific Partnership Is Reached, but Faces Scrutiny in Congress », The New York Times, 6 octobre ; SCHWARZ, J. (2015), « You Can't Read the TPP and You Can't Find Out Who in Congress Has », The Intercept, 13 juin.

${ }^{6}$ DOVERE, E.-I. (2015), « Extreme secrecy eroding support for Obama's trade pact », Politico, 5 avril.

${ }^{7}$ CALMES, J. (2016), « T.P.P. Faces Rough Road in Congress », The New York Times, 2 septembre ; CALMES, J., op. cit., 6 octobre 2016.

8 Office of the United States Trade Representative (2015), «TPP Congressional Timeline », USTradeRepresentative.gov, https://ustr.gov/Congressional-Timeline-TPP. 
s'y étaient progressivement opposés, le président a cherché durant cette période à voir le traité ratifié pour après les élections américaines et avant la mise en place des nouvelles assemblées et du nouvel exécutif américain ${ }^{1}$. Ce délai d'un an, combiné avec les élections américaines ont été déterminant pour expliquer la non-ratification par le Congrès.

En effet, la consultation du Congrès sur ce dossier est arrivée doublement trop tard et dans de mauvaises conditions. Trop tard de prime abord car, les négociations étant déjà achevées, le Congrès et les parlementaires n'ont à priori disposé que d'une très faible marge de manœuvre pour influencer le contenu de l'accord lorsqu'ils ont finalement pu s'inclure dans le processus. Ainsi, l'assemblée n'a pu que débattre sur le bien-fondé ou non des dispositions du document, sans avoir suffisamment le temps d'évaluer l'ensemble des conséquences possibles de la ratification et sans pouvoir discuter en profondeur d'une évolution de certains points avec le Président. Ce qui, étant donné le caractère très impopulaire de l'accord, a rendu la ratification rapide improbable car très risquée au vu de son impact potentiellement négatif, politiquement et économiquement ${ }^{2}$.

L'implication des parlementaires est également arrivée trop tard car, les élections présidentielles et législatives américaines étant en approche, il aurait été possible que le suffrage change les compositions politiques dans les deux chambres législatives, décourageant les implications parlementaires approfondies avant celles-ci. Par la suite, le Congrès a été peu motivé à ratifier le traité après les élections législatives/présidentielles de novembre 2016 comme le Président le souhaitait et avant la mise en place du nouveau Congrès en janvier 2017 de par le caractère illégitime et polémique que cela aurait donné à la ratification. D'autant plus que l'opposition au traité était devenue majoritaire parmi les législateurs ${ }^{3}$. Par ailleurs, il s'est avéré évident que le prochain président, démocrate ou républicain, allait tenter de remettre en cause certaines dispositions du traité.

Il est enfin intéressant de noter que les plus gros rapports du centre de recherche du Congrès sur l'impact du TPP et du TTIP sont parus seulement en 2016, même si des rapports intermédiaires existaient déjà avant ${ }^{4}$. Dans le cas du TPP, la sortie de ce rapport en juin 2016 a montré une fois encore l'implication tardive des parlementaires dans les débats sur le contenu du traité. On peut ainsi y retrouver le constat d'une forme d'impuissance de leur part à influencer l'accord ${ }^{5}$. Néanmoins, le Congrès américain a tenté tout de même d'effectuer son travail de supervision et d'analyse en déterminant si les effets de ratification du traité auraient été favorables ou non aux intérêts américains ${ }^{6}$. Ces derniers étant importants tant en cas de rejet qu'en cas d'adoption pour les États-Unis, le rapport de l'époque a souligné la nécessité de poursuivre plus longuement les travaux d'analyses et d'études sur la mise en œuvre du traité avant d'établir un constat définitif. Cela montre donc que le Congrès américain n'était pas prêt à ratifier le traité à court terme et il est très probable, comme le soulignent beaucoup d'analystes,

\footnotetext{
${ }^{1}$ Ballotpedia (2016), « 2016 presidential candidates on the Trans-Pacific Partnership trade deal », Ballotpedia, https://ballotpedia.org/2016 presidential candidates on the Trans-Pacific Partnership trade deal ; FRASURE, W. G. (2016), « Presidential Election Campaign Dims Prospects for TPP », TheDiplomat, 18 mai ; RITTER, M. (2016), « Approval of TPP in US Congress Seen as Difficult », Learning English, 28 mai.

${ }^{2}$ MCCARTIN, M. et MCCLURE, K. (2016), « What's Next for TPP: Will Congress Ratify in 2016? », global policy watch, 21 janvier; BINDER, K, op. cit.

${ }^{3}$ MARANS, D. (2016), « Progressives Gear Up To Kill TPP In Lame-Duck Congress », The Huffington Post, 19 août ; MCCARTIN, M. et MCCLURE, K., op. cit. ; BINDER, K., op. cit.

${ }^{4}$ WILLIAMS, B.-R. et FERGUSSON, I.-F. (2016), The Trans-Pacific Partnership (TPP): Key Provisions and Issues for Congress (R44489), Washington, Congressional Research Service ; ILIAS AKHTAR, S., JONES, V. et JOHNSON, R. (2016), Transatlantic Trade and Investment Partnership (T-TIP) Negotiations (R43387), Washington, Congressional Research Service.

${ }^{5}$ WILLIAMS Brock R., FERGUSSON Ian F., op. cit.

${ }^{6} \mathrm{Ibid}$.
} 
que l'accord n'aurait pas été ratifié par les parlementaires si Obama avait choisi de le soumettre à un vote de ratification, les forçant à se prononcer après 60 jours de débats ${ }^{1}$.

On peut donc dire que l'impact véritable du Congrès a été de tempérer radicalement le processus de ratification, ce qui a donné le temps au traité de devenir encore davantage un symbole des pertes progressives d'emploi aux États-Unis dans le domaine de la manufacture durant la campagne présidentielle américaine. Cela eu pour conséquences de décourager le Congrès, ainsi que le président Obama, de soumettre le traité à un vote de ratification avant l'élection d'un nouveau président et avant la mise en place des nouveaux députés et sénateurs ${ }^{2}$.

\section{Conclusion}

Peut-on dire au terme de cette analyse qu'il existe une influence claire des parlementaires européens et américains sur la diplomatie menée par les États, ou organisations régionales dans le cas de l'UE, dans la conclusion des traités commerciaux internationaux ?

La réponse est positive. On a pu le constater à plusieurs reprises à travers les différents terrains analysés. Via d'importants travaux de contrôle, d'évaluation et de supervision des négociations par les parlementaires, les institutions législatives peuvent réussir à créer le débat, à rebondir sur des polémiques, à définir leurs priorités et objectifs, à remettre en cause certains points ou décisions des traités négociés, à émettre des demandes et revendications, à discuter avec l'exécutif et au final à se faire entendre par ce dernier si elles en ont suffisamment la volonté. On a ainsi pu découvrir que grâce à ces pressions formelles et informelles de la part des pouvoirs législatifs, mais surtout de la part de la société civile, l'exécutif prend en compte les revendications des parlementaires représentant de cette dernière. Ou tout de moins il tend à le faire. L'augmentation de la transparence des négociations du TTIP et du CETA dans le cas du Parlement européen en est un exemple, de même que la définition des objectifs de négociations du TTIP via le TPA par le Congrès. Il est donc possible qu'une assemblée ait un réel impact sur l'orientation de la diplomatie commerciale de son pays. C'est d'autant plus le cas lorsque l'action ou la légitimité de l'exécutif et de l'accord est contestée ou remise en cause et que le positionnement des chambres législatives est nécessaire pour la ratification du traité, sa mise en œuvre ou son financement.

Le Parlement européen dispose ainsi dans les traités fondateurs de l'UE d'un pouvoir de véto sur les accords commerciaux, ce qui est également le cas du Congrès américain lorsque ce dernier se trouve impliqué via la procédure législative permettant d'adopter un «congressional-executive agreement». Cette dernière tend à être privilégiée par les Américains pour la légitimité démocratique qu'elle confère ou renforce à un accord. L'analyse du TPP a, à ce titre, montré que cette procédure de ratification était grandement compromise si l'exécutif fait cavalier seul dans les négociations sans impliquer suffisamment le Congrès, en l'impliquant trop tardivement ou en ne lui laissant assez de temps pour évaluer le contenu de l'accord final.

Néanmoins il faut cependant émettre des réserves à cette influence. On ne peut ainsi systématiser ces conclusions à l'ensemble des assemblées parlementaires des pays démocratiques et à l'ensemble des accords commerciaux devant être ratifié par le Congrès et le Parlement européen. La marge de manœuvre d'une assemblée législative va dépendre du système dans lequel elle évolue, de sa volonté à participer aux négociations, du contexte politique dans lequel l'accord est négocié et ratifié et enfin elle va dépendre de la complaisance de l'exécutif vis-à-vis du respect strict ou souple des règles de processus de négociation. L'implication d'un parlement n'est donc pas systématique à chaque État et sur chaque sujet. Si

\footnotetext{
${ }^{1}$ FRASURE, W. G., op. cit.

${ }^{2}$ Ibid.; MARANS, D, op. cit.; MORTIMER, C. (2016), « Trans-Pacific trade deal will not be passed by Congress before inauguration, White House admits », TheIndependent, 13 novembre.
} 
le Parlement européen a gagné sa participation dans les négociations commerciales du TTIP et du CETA grâce à une interprétation extensive de ses compétences et grâce à la bonne volonté de l'exécutif européen au vu du besoin croissant de conférer une légitimité démocratique aux accords commerciaux, il n'existe cependant dans les faits aucune règle formelle qui impose de pérenniser cette implication à un tel degré pour chaque accord commercial. Il serait donc possible que pour d'autres accords son influence soit plus limitée s'il n'est pas motivé à s'impliquer davantage que le nécessaire. Cela a d'ailleurs failli être le cas pour le CETA comme on a pu le voir.

À l'inverse, aux États-Unis l'exécutif est obligé légalement et juridiquement de donner une influence au Congrès dans la négociation du traité lorsque la procédure de «fast track » est initiée via la "trade promotion autority ». Néanmoins, la présence de cette règle ne veut pas dire pour autant que l'exécutif va l'appliquer au mieux. Ainsi l'implication du Congrès a été mise en œuvre quelques mois à peine avant que les négociations du TTP soient conclues, sans que l'une ou l'autre chambre législative ait pu avoir accès dans son ensemble et de manière approfondie au contenu du traité. On a pu constater que l'assemblée a été incapable, de par le mandat qu'elle avait accordé précédemment, de modifier par elle-même le contenu du traité et qu'elle n'avait par ailleurs, une fois une certaine période de temps écoulée, aucune motivation à s'impliquer davantage dans celui-ci pour qu'il soit modifié ou ratifié ; contrairement au Parlement européen qui a réussi à faire entendre ses revendications après la conclusion des négociations sur le CETA. La différence ici est que l'assemblée désirait majoritairement que le traité soit ratifié à terme, mais avec des modifications et clarifications. Ce qui fut le cas. On peut se demander dans le cas du Congrès américain si ce dernier aurait pu de la même manière réclamer certaines modifications ou interprétations sine qua non à la ratification du traité, s'il avait obtenu plus de temps pour l'analyser, s'il avait été inclus plus tôt dans les négociations et si cette assemblée avait eu effectivement la volonté de ratifier l'accord final. Ce fut par exemple le cas en janvier 2020 pour l'accord renégociant la zone de libre-échange nord-américaine où le Président a soumis une nouvelle version de l'accord après avoir négocié avec les parlementaires américains ${ }^{1}$. Le contexte et la mobilisation du pouvoir législatif sont donc deux données essentielles.

Par ailleurs, l'on a pu voir avec le CETA que l'influence d'une assemblée est beaucoup plus limitée si le Parlement ne communique pas et si l'accord ne suscite pas d'intérêt de la part du public ou reste relativement ignoré de celui-ci. A contrario, dans le cas présent, à partir du moment où la polémique a éclaté, le Parlement a finalement remis en cause une partie du contenu de l'accord négocié et exigé des réformes.

Dès lors, si l'on peut affirmer qu'il y a une implication croissante des assemblées dans les matières internationales, cela se fait à des échelles différentes en fonction des différents États concernés. Si on a pu voir une participation importante du Parlement européen dans les négociations commerciales étudiées, cette implication s'est faite de manière plus limitée pour les cas ciblant le Congrès des États-Unis. Celui-ci a bien fixé des objectifs et critères à faire remplir et a pu utiliser son pouvoir de supervision, mais son impact concret reste lui beaucoup moins visible dans le processus de négociations et sur le contenu des traités que dans le cas du Parlement européen car, contrairement à ce dernier, les parlementaires américains n’ont pas su tirer profit de l'impopularité des accords pour obtenir davantage de transparence ou d'implication du fait de la nature de la «fast track procedure » ou et de leur manque de motivation à voir un accord aboutir. Si l'on ne peut affirmer que cette situation est généralisable à l'ensemble des accords commerciaux américains, de par le faible nombre de cas analysés, il n'en reste pas moins une réalité dans les cas présents.

\footnotetext{
${ }^{1}$ COCHRANE, E. et SWANSON, A. (2019), « Trump Aides and Democrats Agree on Trade Pact With Mexico and Canada », The New York Times, 10 décembre.
} 
Par ailleurs, malgré cette participation du pouvoir législatif aux affaires de politique extérieure commerciale, on ne peut affirmer que les parlementaires sont devenus des diplomates. La meilleure manière pour une assemblée législative d'influencer l'exécutif n'est pas d'agir au niveau international, en dialoguant avec d'autres parlements ou en tentant d'établir des contacts diplomatiques, mais bien en soumettant ou en reprenant à son compte certaines pressions sur les négociateurs au niveau national, tout en appliquant ses compétences internes via les commissions parlementaires ou les débats en interne. On a pu voir le faible écho des relations interparlementaires américo-européenne ou canado-européenne sur le TTIP et le CETA, contrairement aux auditions parlementaires et aux déclarations ou avis des deux assemblées sur l'avancée des négociations. Finalement, l'utilisation de l'arme du droit de véto parlementaire sur un accord, élément de pouvoir le plus important des assemblées, reste un outil législatif à visée national, mais dont l'impact se veut international.

Il convient dès lors de tenir compte de l'influence des assemblées législatives sur la diplomatie des États en considérant l'ensemble des éléments venant d'être décrits dans la phase finale de cette analyse. Il est difficile d'affirmer que les tendances identifiées dans cette analyse se maintiendront, augmenteront ou diminueront pour les futures négociations commerciales internationales car, d'une part, la politique internationale est toujours soumise à de nouveaux développements, et d'autre part, la capacité d'apprentissage et d'adaptation des parlementaires sur ces questions est importante et pourrait évoluer de plusieurs manières par la suite. Les perspectives d'analyse restent donc très nombreuses dans le cadre de l'étude du rôle des assemblées parlementaires en politique commerciale extérieure et leur nombre ne manquera pas d'augmenter dans les années à venir.

\section{Bibliographie}

ALEXOAEI, A. et COJANU, V. (2017), « Negotiating the Transatlantic deal: focus on the EU's domestic constraints », CES Working Papers, Centre for European Studies, Alexandru Ioan Cuza University, vol. 9, $\mathrm{n}^{\circ}$ 3, pp. 233-254.

ARC (2014), « Transatlantic Dialogue between legislators on TTIP in European Parliament », Arc 2020, 21 juillet 2014, www.arc2020.eu/front/2014/07/transatlantic-dialogue-betweenlegislators-on-ttip-in-the-european-parliament (consulté en août 2019).

Ballotpedia (2016), « 2016 presidential candidates on the Trans-Pacific Partnership trade deal », Ballotpedia, https://ballotpedia.org/2016_presidential_candidates_on_the_TransPacific_Partnership_trade_deal (consulté en août 2019).

BEAUSSIER, A.-L. et BENJABALLAH, S. (2014), «Comparer le Parlement européen et le Congrès américain. Un état des lieux théorique et méthodologique », Revue internationale de politique comparée, vol. 21, $\mathrm{n}^{\circ} 3$, pp. 9-30.

BERGBOWER, M. L. et SCHMITT, C. (2015), « Congressional Activism in the Foreign Policy Arena: A Case Study of the 1994 Rwandan Genocide », Democracy and Security, vol. 11, n 4 , pp. 353-372.

BINDER, K. (2017). Briefing : From TPP to new trade arrangements in the Asia-Pacific region(PE 603.953), Bruxelles, European Parliament Research Service, https://www.europarl.europa.eu/RegData/etudes/BRIE/2017/603953/EPRS_BRI(2017)60395 3_EN.pdf (consulté en août 2019). 
BRICART, V. (2017), «Le Congrès américain et les pouvoirs de guerre : de la théorie à la pratique », CEFIR Working Paper, n 3, pp. 1-19.

CALMES, J. (2015), « Trans-Pacific Partnership Is Reached, but Faces Scrutiny in Congress », The New York Times, 6 octobre.

CALMES, J. (2016), «T.P.P. Faces Rough Road in Congress », The New York Times, 2 septembre.

COCHRANE, E. et SWANSON, A. (2019), «Trump Aides and Democrats Agree on Trade Pact With Mexico and Canada », The New York Times, 10 décembre.

Committee on International Trade. (2016). Mission Report: Following the ad-hoc delegation to Canada (Ottawa and Montreal) from 21 to 23 March 2016 ( CR\1089805EN.doc PE578.851v01-00), Bruxelles, European Parliament, https://www.europarl.europa.eu/cmsdata/102098/Canada_Mission_Report.pdf (consulté en août 2019).

Committees (2019), «Hearings», European Parliament Committees, https://www.europarl.europa.eu/committees/en/indexsearch.html?term=9\&query=TTIP (consulté en août 2019).

COSTA, O. (2014), « Que peut le Parlement européen ? », Pouvoirs, vol. 149, n 2, pp. 77-89. COURMONT, B. (2001), «Le Congrès et son poids dans la formulation de la politique étrangère américaine », Revue internationale et stratégique, vol. 42, n 2, pp. 87-97.

Damen, D. (2019), «L’Union européenne et ses partenaires commerciaux », Fiches thématiques l'Union européenne, novembre 2019, http://www.europarl.europa.eu/factsheets/fr/sheet/160/1-union-europeenne-et-ses-partenairescommerciaux (consulté en août 2019).

DAVID, C-P. (éd.) (2015), La politique étrangère des Etats-Unis : fondements, acteurs,

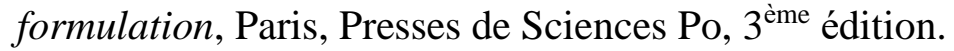

DAVIES BOREN, Z. (2015), « TTIP controversy: Secret trade deal can only be read in secure 'reading roo' in Brussels », TheIndependent, 4 août.

DEL MONTE, M. et PUCCIO, L. (2016). Rôle du Congrès des États-Unis dans les accords commerciaux, La procédure accélérée "Fast-Track procedure "(PE 577.999), Bruxelles, European Parliament Research Service https://www.europarl.europa.eu/RegData/etudes/IDAN/2016/577999/EPRS_IDA(2016)57799 9_FR.pdf (consulté en août 2019).

DOVERE, E.-I. (2015), « Extreme secrecy eroding support for Obama's trade pact », Politico, 5 avril.

DUCOURTIEUX, C. (2016), « La galaxie hétéroclite des anti-TTIP », Lemonde, 16 septembre.

DUCOURTIEUX, C. (2016), «Les autorités peinent à convaincre les opinions publiques », Lemonde, 30 avril. 
DUCOURTIEUX, C. (2018), « Europe : Bruxelles contrainte à la transparence sous la pression des opinions publiques », Lemonde, 19 mai.

ERMERT, M. (2015), «TPP Heads Into Ratification Game », Intellectual Property Watch, 6 octobre.

European Commission (2014), «Online public consultation on investment protection and investor-to-state dispute settlement (ISDS) in the Transatlantic Trade and Investment Partnership Agreement (TTIP)», europa.eu, juillet 2014, http://trade.ec.europa.eu/consultations/index.cfm?consul_id=179 (consulté en août 2019).

European Commission (2015), «Dans un souci de transparence, la Commission européenne publie des textes juridiques concernant le TTIP», europa.eu, 7 janvier 2015, https://europa.eu/rapid/press-release_IP-15-2980_fr.htm (consulté en août 2019).

European Commission (2015), «Présentation ce jour d'un rapport sur la consultation concernant la protection des investissements dans les négociations commerciales UE-EtatsUnis », europa.eu, 13 janvier 2015, https://europa.eu/rapid/press-release_IP-15-3201_fr.htm (consulté en août 2019).

European Commission (2016), «EU negotiating texts in TTIP », europa.eu, 14 juillet 2016, http://trade.ec.europa.eu/doclib/press/index.cfm?id=1230 (consulté en août 2019).

European Commission. (2015). Online public consultation on investment protection and investor-to-state dispute settlement (ISDS) in the Transatlantic Trade and Investment Partnership Agreement (TTIP) (13.1.2015 SWD (2015) 3 final), Bruxelles, http://trade.ec.europa.eu/doclib/docs/2015/january/tradoc_153044.pdf (consulté en août 2019).

European Parliament (2010), «SWIFT: European Parliament votes down agreement with the US », europa.eu, 11 février 2010, https://www.europarl.europa.eu/sides/getDoc.do?pubRef=//EP//TEXT+IM-PRESS+20100209IPR68674+0+DOC+XML+V0//EN (consulté en août 2019).

European Parliament (2014), «Politics \& Policies - April 2014 », europa.eu, 10 avril 2014, https://www.europarl.europa.eu/unitedstates/en/eplo-news/politics-policies-april-2014 (consulté en août 2019).

European Parliament (2017), «CETA: MEPs back EU-Canada trade agreement », europa.eu, 2 février 2017, http://www.europarl.europa.eu/news/en/press-room/20170209IPR61728/cetameps-back-eu-canada-trade-agreement (consulté en août 2019).

European Parliament (2019), «Bureaux de liaison dans votre pays », europa.eu, http://www.europarl.europa.eu/at-your-service/fr/stay-informed/liaison-offices-in-yourcountry (consulté en août 2019).

European Parliament (2019), «European Parliament Committees », europa.eu, https://www.europarl.europa.eu/committees/en/parliamentary-committees.html (consulté en août 2019). 
European Parliament \& US House of Representatives. (2013). $74^{\text {TH }}$ Inter-parliamentary meeting: Transatlantic Legislators' Dialogue (Joint Statement of 2013), Bruxelles, https://www.europarl.europa.eu/cmsdata/122911/TLD\%20June\%202013.pdf (consulté en août 2019).

European Parliament \& US House of Representatives. (2014). $75^{\text {TH }}$ Inter-parliamentary meeting: Transatlantic Legislators' Dialogue (Joint Statement of 2014), Bruxelles, https://www.europarl.europa.eu/cmsdata/122912/TLD\%20March\%202014.pdf (consulté en août 2019).

European Parliament \& US House of Representatives. (2015). $77^{T H}$ Inter-parliamentary meeting: Transatlantic Legislators' Dialogue (2 ${ }^{\text {nd }}$ Joint Statement of 2015), Bruxelles, https://www.europarl.europa.eu/cmsdata/122148/Joint_Statement_EU-US_IPM_4-

5_Nov_2015.pdf (consulté en août 2019).

European Parliament Delegation for Relations with Canada (2019), « Welcome from the former Chair », europa.eu, https://www.europarl.europa.eu/delegations/en/d-ca/home (consulté en août 2019).

European Parliament Delegation for Relations with Canada. (2014). Delegation for Relations with Canada $36^{\text {th }}$ EP-Canada Inter-Parliamentary Meeting Brussels 13 November 2014 (Mission Report of 2014), Bruxelles, European Parliament, https://www.europarl.europa.eu/cmsdata/124161/Report\%2036th\%20EP-Canada\%20IPM.pdf (consulté en août 2019).

European Parliament Delegation for Relations with Canada. (2017). Delegation for Relations with Canada $37^{\text {th }}$ EU-Canada Inter-Parliamentary Meeting Ottawa, Quebec and Montreal 1620 May 2016 (Mission Report of 2016), Bruxelles, European Parliament, https://www.europarl.europa.eu/cmsdata/124140/Mission\%20report.pdf (consulté en août 2019).

European Parliament Liaison Office in Washington DC (2013), « Transatlantic Trade and Investment Partnership: MEPs gauge 'increasingly positive mood' in Washington, outline key EU interests », europa.eu, 11 april 2013, www.europarl.europa.eu/us/en/news_events/news/news_2013/news_apr_2013/ttip_eu_interes ts.html (consulté en août 2019).

European Parliament, «EU trade and investment agreement negotiations with the US », Resolution, P7_TA (2013)0227, 23 May 2013.

European Parliament, «European Parliament resolution of 8 June 2011 on EU-Canada trade relations », Resolution, P7_TA (2011)0257, 8 June 2011.

European Parliament, «European Parliament's Recommendations to the European Commission on the Negotiations for the Transatlantic Trade and Investment Partnership (TTIP) », Resolution, P8_TA(2015)0252, 8 July 2015.

European Parliament, «TTIP: Challenges and Opportunities for the Internal Market», Joint Public Hearing Committee on the Internal Market and Consumer Protection Committee on 
International Trade,

http://www.europarl.europa.eu/committees/en/inta/eventshearings.html?id=20150210CHE00121, 24 February 2015.

European Parliament, «TTIP: What's in it for Europeans? », Public Hearing Committee on International Trade, DV $1053409 E N . d o c, 18$ March 2015.

European Parliament. (2019). EU-Canada comprehensive economic and trade agreement (CETA) (Legislative Train $\mathrm{n}^{\circ} 07.2019$ 6A), Bruxelles, https://www.europarl.europa.eu/legislative-train/api/stages/report/07-2019/theme/a-balancedand-progressive-trade-policy-to-harness-globalisation/file/ceta (consulté en août 2019).

FERGUSSO, I.-F. (2015). Trade Promotion Authority (TPA) and the Role of Congress in Trade Policy (RL33743), Washington, Congressional Research Service, https://fas.org/sgp/crs/misc/RL33743.pdf (consulté en août 2019).

FORTMANN, M. (dir.) et MARTIN P. (dir.) (2013), Le système politique américain, Montréal,

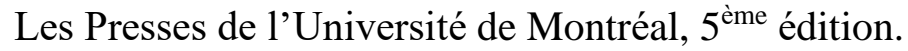

FRASURE, W. G. (2016), «Presidential Election Campaign Dims Prospects for TPP», TheDiplomat, 18 mai.

FROMAGE, D. (2018), «The European Parliament in the post-crisis era: an institution empowered on paper only?», Journal of European Integration, vol. 40, n 3, pp. 281-294.

Giovenco, A. (2013), « Chief U.S. and EU Negotiators Brief Congressional Staff on the First Round of TTIP Negotiations », Tradewinds, the official blog of the United States Trade Representative, juillet 2013, https://ustr.gov/about-us/policy-offices/pressoffice/blog/2013/july/negotiators-brief-hill-ttip (consulté en août 2019).

HUBER, K. (2015), «The European Parliament as an actor in EU border policies: its role, relations with other EU institutions, and impact », European Security, vol. 24, n 3, pp. 420437.

ILIAS AKHTAR, S. et JONES, V. (2014). Transatlantic Trade and Investment Partnership (TTIP) Negotiations (R43387), Washington, Congressional Research Service, https://fas.org/sgp/crs/row/R43387.pdf (consulté en août 2019).

ILIAS AKHTAR, S., JONES, V. et JOHNSON, R. (2016). Transatlantic Trade and Investment Partnership (T-TIP) Negotiations (R43387), Washington, Congressional Research Service, https://nationalaglawcenter.org/wp-content/uploads//assets/crs/R43387.pdf (consulté en août 2019).

JANČIĆ, D. (2015), «Transatlantic Regulatory Interdependence, Law and Governance: The Evolving Roles of the EU and US Legislatures », Cambridge Yearbook of European Legal Studies, vol. 17, pp. 334-359.

JANČIĆ, D. (2016), « The Role of the European Parliament and the US Congress in Shaping Transatlantic Relations: TTIP, NSA Surveillance, and CIA Renditions », Journal of Common Market Studies, vol. 54, n 4, pp. 896-912. 
JANČIĆ, D. (2017), «TTIP and legislative-executive relations in EU trade policy », West European Politics, vol. 40, n 1, pp. 202-221.

KANDEL, M. (2016), «Le rôle du Congrès américain dans le processus de décision en politique étrangère : les accords de Dayton (1995) », Critique internationale, vol. 71, $\mathrm{n}^{\circ}$ 2, pp. 109-127.

KEUKELEIRE, S. et DELREUX, T. (2014), The Foreign Policy of the European Union, Londres, MacMillan Education UK, 2 ème édition.

LAROCCO, L. A. (2017), «Wilbur Ross says he's 'open to resuming' talks on mega-trade deal with Europe », $C N B C, 30$ mai.

Malmström, C. (2014), «The Trade Commissioner and Parliament: A Vital Relationship », europa.eu, 3 décembre 2014, https://europa.eu/rapid/press-release_SPEECH-14-2348_en.htm (consulté en août 2019).

MARANS, D. (2016), « Progressives Gear Up To Kill TPP In Lame-Duck Congress », The Huffington Post, 19 août.

MCCARTIN, M. et MCCLURE, K. (2016), «What's Next for TPP: Will Congress Ratify in $2016 ?$ ?, global policy watch, 21 janvier.

MORTIMER, C. (2016), « Trans-Pacific trade deal will not be passed by Congress before inauguration, White House admits », TheIndependent, 13 novembre.

NEW, W. (2012), «US Congress Members Demand Access To TPP; ACTA Criticised In Australia », Intellectual Property Watch, 27 juillet.

Office of the United States Trade Representative (2015), «TPP Congressional Timeline », USTradeRepresentative.gov, https://ustr.gov/Congressional-Timeline-TPP (consulté en août 2019).

PASQUARIELLO MARIONO, K. L. et THODORO LUCIONA, B. (2018), "The parliamentarization of EU trade policy: unveiling the European Parliament's involvement in EU-MERCOSUR trade negotiations », European Politics and Society, vol. 20, $\mathrm{n}^{\circ}$ 5, pp. 591-608.

PUCCIO, L. (2016). Négociations UE/États-Unis sur le PTCI, Vue d'ensemble des questions en suspens (PE 586.606), Bruxelles, European Parliament Research Service, https://www.europarl.europa.eu/RegData/etudes/IDAN/2016/586606/EPRS_IDA(2016)58660 6_FR.pdf (consulté en août 2019).

RIDDERVOLD, M. et ROSÉN, G. (2016), « Trick and treat: how the Commission and the European Parliament exert influence in EU foreign and security policies », Journal of European Integration, vol. 38, $\mathrm{n}^{\circ}$ 6, pp. 687-702.

RIPOLL SERVENT, A. (2014), «The role of the European Parliament in international negotiations after Lisbon », Journal of European Public Policy, vol. 21, n 4, pp. 568-586. 
RITTER, M. (2016), «Approval of TPP in US Congress Seen as Difficult », Learning English, 28 mai.

ROEDERER-RYNNING, C. (2017), «Parliamentary assertion and deep integration: the European parliament in the CETA and TTIP negotiations », Cambridge Review of International Affairs, vol. 30, n ${ }^{\circ}$ 5-6, pp. 507-526.

ROEHRIG, T. et WESSEL, L. A. (2011), « Congress and U.S.-North Korean Relations: The Role of the Entrepreneur », Asian Affairs: An American Review, vol. 38, n 2, pp. 89-108.

ROSÉN, G. (2017), « The impact of norms on political decision-making: how to account for the European Parliament's empowerment in EU external trade policy », Journal of European Public Policy, vol. 24, n ${ }^{\circ}$ 10, pp. 1450-1470.

SCHÖLLMANN, W. (2017). Briefing International Agreements in Progress: Comprehensive Economic and Trade Agreement (CETA) with Canada (PE 595.895), Bruxelles, European Parliament Research Service, https://www.europarl.europa.eu/RegData/etudes/BRIE/2017/595895/EPRS_BRI(2017)59589 5_EN.pdf (consulté en août 2019).

SCHWARZ, J. (2015), « You Can't Read the TPP and You Can't Find Out Who in Congress Has », The Intercept, 13 juin.

STEHRER, R. et STÖLLINGER, R. (2016), « CETA and TTIP: same-same but different? », Vienna Institute for International Economic Studies, 6 septembre.

THE GUARDIAN (2013), «WikiLeaks publishes secret draft chapter of Trans-Pacific Partnership », TheGuardian, 13 novembre.

Traité sur l'Union Européenne (TUE), signé à Maastricht le 7 février 1992, modifié le 13 décembre 2007.

Traité sur le Fonctionnement de l'Union européenne (TFUE), signé à Rome le 25 mars 1957, modifié le 13 décembre 2007.

Transatlantic Legislators' Dialogue (2019), « Transatlantic Legislators' Dialogue : Welcome », europa.eu, https://www.europarl.europa.eu/intcoop/tld/default_en.htm (consulté en août 2019).

United Sates Senate (2019), «Hearings \& Meetings », senate.gov, https://www.senate.gov/committees/hearings_meetings.htm (consulté en août 2019).

United States Constitution, signée à Philadelphie le 17 septembre 1787, modifiée le 7 mai 1992.

United States Senate Committee on Finance (2016), «Hatch, Brady Send Letter to USTR as TTIP Negotiations with EU Resume », US Senate Committee on Finance, 3 octobre 2016, https://www.finance.senate.gov/chairmans-news/hatch-brady-send-letter-to-ustr-as-ttipnegotiations-with-eu-resume (consulté en août 2019).

US Congress, «Bipartisan Congressional Trade Priorities and Accountability Act of 2015 », Public Law, 114-26 (114 $\left.{ }^{\text {th }}\right)$, Congress, 29 June 2015. 
US House of Representatives Ways and Means' committee (2015), « Hearing on Trade », Way $\&$ Means https://waysandmeans.house.gov/legislation/hearings/hearing-trade (consulté en août 2019).

US House of Representatives, «Expanding American Trade with Accountability and Transparency », Hearings Before the Committee of Ways and Means, 114-FC04 $\left(114^{\text {th }}\right)$, Congress, 22 April 2015.

US House of Representatives, «Expressing the sense of the House of Representatives that the United States and the European Union should pursue a Transatlantic Trade and Investment Partnership », Resolution, H.Res.76 $\left(113^{\text {th }}\right)$, 15 February 2013.

US House of Representatives, «U.S. Trade Policy Agenda », Hearings Before the Committee of Ways and Means, 114-FC02 (114 $\left.{ }^{\text {th }}\right), 27$ January 2015.

VINCENTI, D. (2016), «Parliament committee gives CETA thumbs down», euractiv, 9 décembre.

Wikileaks (2014), «Updated Secret Trans-Pacific Partnership Agreement (TPP) - IP Chapter (second publication) », wikileaks, 16 octobre 2014, https://wikileaks.org/tpp-ip2/ (consulté en août 2019).

WILLIAMS, B.-R. et FERGUSSON, I.-F. (2016). The Trans-Pacific Partnership (TPP): Key Provisions and Issues for Congress (R44489), Washington, Congressional Research Service, https://fas.org/sgp/crs/row/R44489.pdf (consulté en août 2019).

WILLIAMS, B.-R. et FERGUSSON, I.-F. (2018). TPP: Overview and Current Status. (IF10000 Version $\mathrm{n}^{\circ} 28$ ), Washington, Congressional Research Service, https://crsreports.congress.gov/product/pdf/IF/IF10000/28 (consulté en août 2019).

WINZEN, T. (2011), « Technical or Political? An Exploration of the Work of Officials in the Committees of the European Parliament », The Journal of Legislative Studies, vol. 17, $\mathrm{n}^{\circ} 1$, pp. 27-44. 